\title{
The possibility of studying polarization observables in reactions of the production of pions by polarized beams of protons and deuterons at the JINR LHEP
}

\author{
Anatoliy Litvinenko ${ }^{1, *}$ \\ ${ }^{1}$ Joint Institute for Nuclear Research, Dubna, Moscow region, 141980, Russia, \\ University Dubna, Dubna, Moscow region, 141980, Russia
}

\begin{abstract}
Along with studying the properties of strongly excited hadronic matter at high densities of the baryon charge, studies with polarized protons and deuterons are planned at the LHEP accelerator complex (see [1-3]). The purpose of this note is to analyze the possibility of obtaining new experimental data, based on the presence of beams of polarized protons and deuterons. In addition, the use of extracted beams of tensor-polarized deuterons to study the structure of the deuteron at small internucleon distances is discussed.
\end{abstract}

\section{The study of the vector analyzing power of the reaction of pion production by vector polarized deuterons, protons and neutrons on the MPD facility}

Measurement of the vector analyzing power $A_{y}$ of reaction of pion production by polarized protons

$$
p \uparrow+p(A)=\pi^{ \pm}+X
$$

is of interest because it helps in understanding the proton spin structure. In the report for vector analyzing power, along with $A_{y}$ the designation $A_{N}$ will be used. Asymmetry $A_{N}$ as a function of the Feynman variable $\left(x_{F}=p / \sqrt{s}\right)$ has similar behavior at high and intermediate energies (see [4-7]), as shown in Fig. 1.

Let us dwell on the discussion of the possibility of studying the asymmetry of pion production in the scattering of vector-polarized protons and deuterons using Multi Purpose Detector (MPD) Fig. 2.

Simulation was performed for the TPC and barrel part of the time-of-flight system. A solenoidal magnetic field with an induction of $0.5 \mathrm{~T}$ was assumed. The pion production cross section was chosen following [10]. The simulation was carried out for three polarization modes:

$$
p_{+}=0.7 ; \quad p_{0}=0 ; \quad p_{-}=-0.7
$$

The analysis was performed for a particle from the fragmentation region one of the colliding beams $x_{F}=p_{\pi, \|} / p_{\pi, \max } \geq 0.5$, where $p_{\pi, \|}$ is the longitudinal momentum of the pion,

\footnotetext{
*e-mail: alitvin@jinr.ru
} 


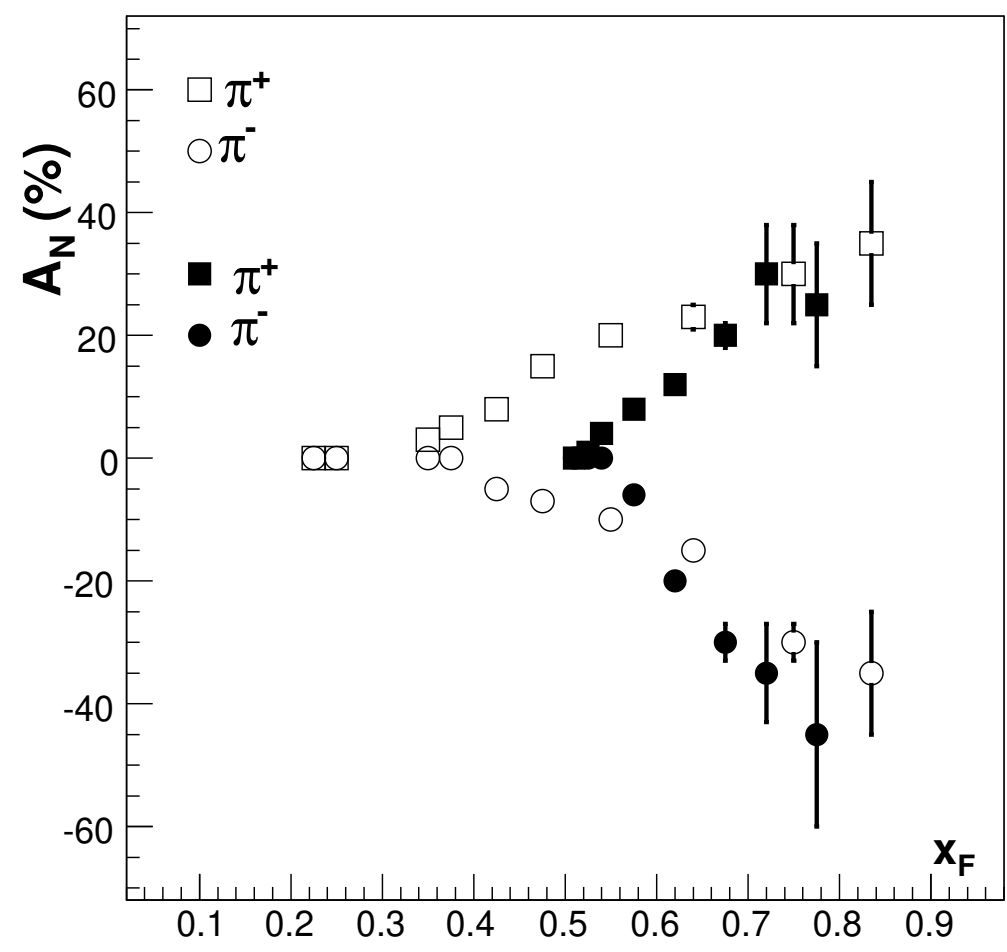

Figure 1. Experimental data on the asymmetry of the production of charged pions at low $p_{b}=$ $21.6 \mathrm{GeV} / \mathrm{c}$ (open symbols), $\sqrt{S_{p, p}}=6.4 \mathrm{GeV}$ (see [7]) and high $p_{b}=200 \mathrm{GeV} / \mathrm{c}$ (closed symbols), $\sqrt{S_{p, p}}=19.4 \mathrm{GeV}[5]$ energies. (data taken from [7])

$p_{\pi, \max }$ is the maximum possible momentum of the pion in the reaction (1). Preliminary estimates show that with the designed parameters NICA and MPD, it is possible to obtain the value of the asymmetry coefficient $A_{N}=(d \sigma \uparrow-d \sigma \downarrow) /(d \sigma \uparrow+d \sigma \downarrow)$ with an accuracy of about $5 \%$ (see Fig. 2). With vector polarization, the cross section is written as:

$$
E d \sigma / d \vec{p}=d \sigma_{0}\left(x_{F}, p_{T}, s\right)\left(1+A_{N}\left(x_{F}, p_{T}, s\right) P \cos \varphi\right) .
$$

The result for the experimental data, which in this article is taken from the simulation, is written through the normalized distributions over the azimuth angle $\varphi$ (measured from the axis of the spin direction perpendicular to the beam direction):

$$
n_{i}\left(\varphi_{j}\right)=\left(\Delta N_{i}\left(\varphi_{j}\right) / \Delta \varphi\right) L_{i}
$$

where $i=+, 0,-$ is the polarization mode, $\Delta N_{i}$ is the total number of pions in polarization mode in the interval $\Delta \varphi$ in at the angle $\varphi_{j}$ and $L_{i}$ is the integral luminosity for the corresponding polarization. The asymmetry through such distributions is expressed by the following equation:

$$
A_{N}=\frac{3}{P} \cdot \frac{\left\langle n_{+} \cos \varphi\right\rangle-\left\langle n_{-} \cos \varphi\right\rangle}{\left\langle n_{-}\right\rangle+\left\langle n_{0}\right\rangle+\left\langle n_{+}\right\rangle}
$$

With notation:

$$
\left\langle n_{i}\right\rangle=\sum_{j=1}^{N_{\varphi}} n_{i}\left(\varphi_{j}\right) ; \quad\left\langle n_{i} \cos \varphi\right\rangle=\sum_{j=1}^{N_{\varphi}} n_{i}\left(\varphi_{j}\right) \cos \varphi_{j}
$$




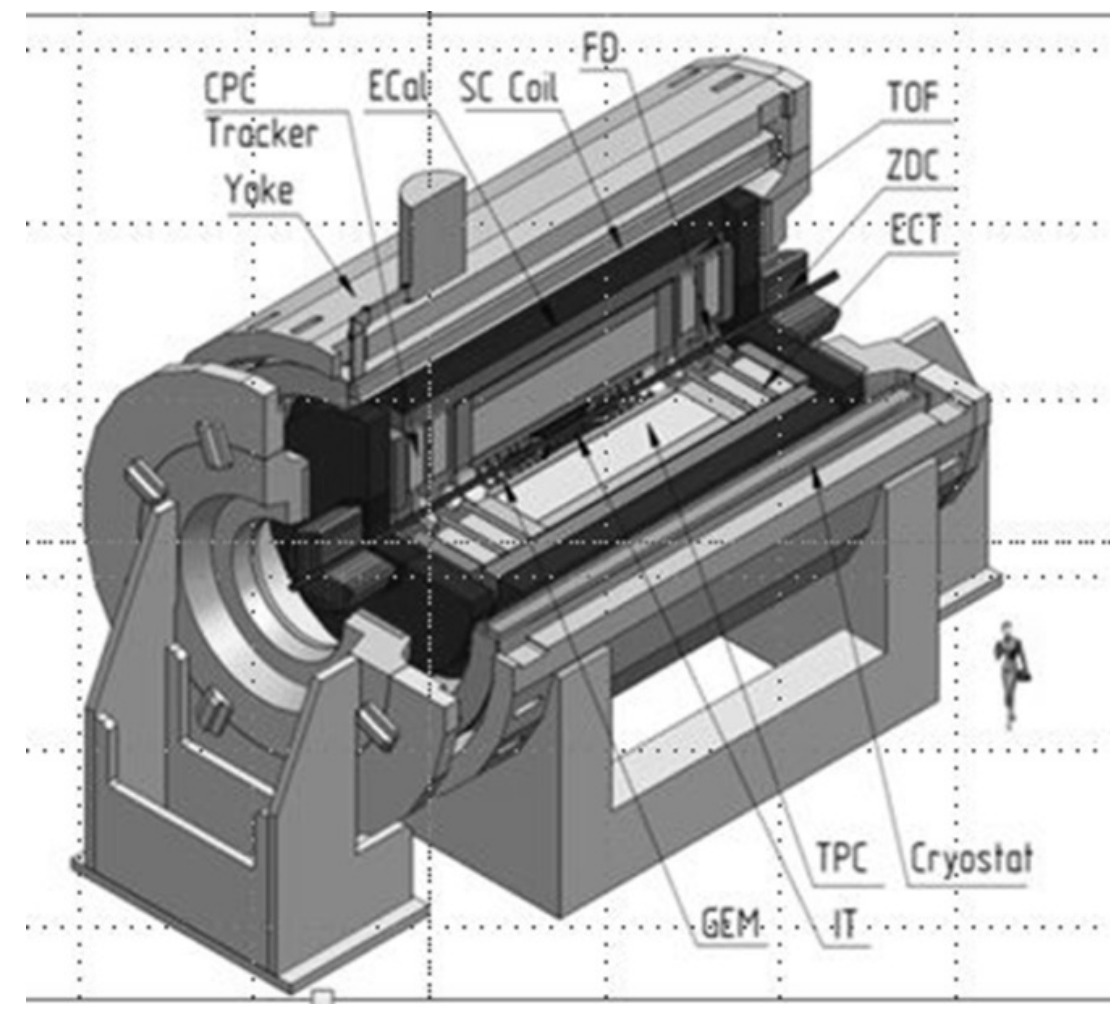

Figure 2. General view of the Multi Purpose Detector (MPD), with the denotation of the main subsystems of ther detector [9]

The results of the simulation of the experimental data for asymmetry are shown in Fig. 3. The pion production cross section was calculated using the approximations from [10], and the asymmetry value was calculated using the model from [11].

Let us dwell on the possibilities of obtaining the asymmetry of the production of pions by polarized neutrons. This is possible because the NICA plans the studies with vector polarized deuterons [3]. The deuteron of the $\operatorname{spin} S_{d}=1$ consists of a mixture of states with the orbital angular momenta $l=0$ ( $S$-wave) and $l=2$ ( $D$-wave) (see, for example, [12]). In all common accepted deuteron models, the contribution of the $D$-wave does not exceed 5\%. If we neglect the $D$-wave contribution, then the deuteron can be represented as a state of a neutron and a proton having spins directed along a spin of deuteron.

Within the framework of such an assumption with the known asymmetries of pion production by deuterons $A_{N}(d)$ and protons $A_{N}(p)$, we can obtain the asymmetry of pion production by polarized neutrons:

$$
A_{N}(n)=\frac{\sigma_{0}(d) A_{N}(d)-\alpha \sigma_{0}(p) A_{N}(p)}{\sigma_{0}(d)-\alpha \sigma_{0}(p)} .
$$

Here $\sigma_{0}(d)$ and $\sigma_{0}(p)$ are the pion production cross sections of unpolarized deuterons and protons, respectively, and $\alpha=0.85$ is the shading factor calculated in the Glauber approximation, which describes the cross sections for the interaction of deuterons with nuclei with good accuracy (see [13]). Therefore, having experimental data on the asymmetry of pion production by protons and vector polarized deuterons, one can obtain the asymmetry of pion 

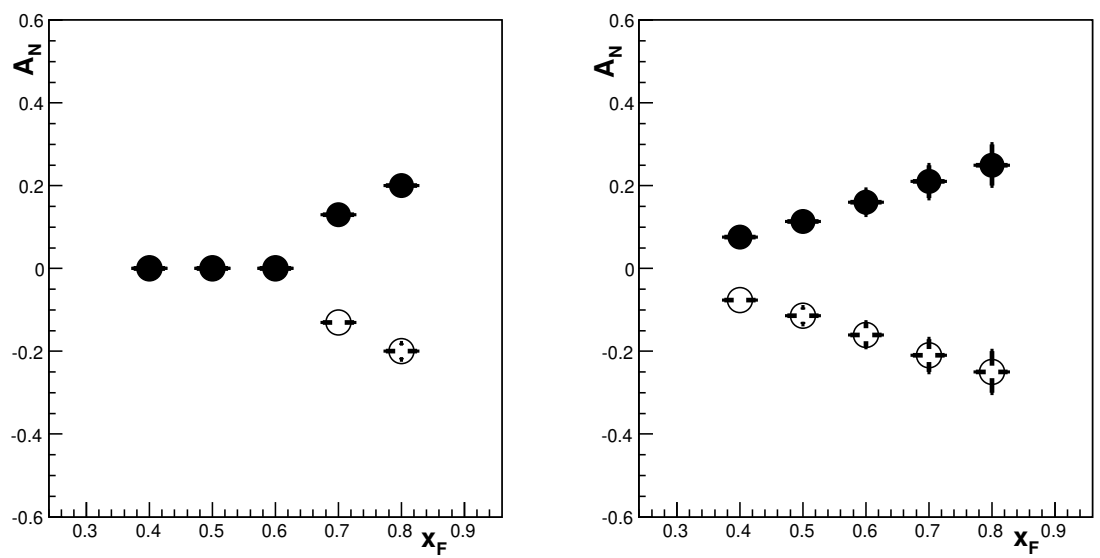

Figure 3. The value of asymmetry $\left(A_{y} \equiv A_{N}\right)$ can be obtained in a month of operation of MPD on a beam of polarized protons with a luminosity of $10^{31} \mathrm{~cm}^{-2} \mathrm{~s}^{-1}$ (an order of magnitude less than the design) and energy $\sqrt{s}=4 \mathrm{GeV}$ (left $p_{T}>1.4 \mathrm{GeV} / \mathrm{c}$, right $p_{T}>1.6 \mathrm{GeV} / \mathrm{c}$ )

production by polarized neutrons. It should be noted that to obtain the asymmetry of pion production by polarized neutrons, it is necessary to know the absolute luminosity. This is evident from (7) because without absolute luminosity it is impossible to obtain cross sections for the production of pions by protons and deuterons.

\section{The study of polarization observed in the reaction of pion production by tensor-polarized deuterons in the experiment with a fixed target}

The study of polarization observables in the pion production reaction by tensor-polarized deuterons makes it possible to clarify the relative contribution of $S$ and $D$ waves in the deuteron at large $(\geq 0.2 \mathrm{GeV} / \mathrm{c})$ relative momentum. For this, the pion must be registered in the region of deuteron fragmentation in the subthreshold (inaccessible in $p+p$ collisions) kinematic region (cumulative region). The calculations in the impulse approximation show that the value of the tensor analyzing power $T_{20}$ is sensitive to the relative contribution of $S$ and $D$ waves and weakly depends on the amplitude of pion production [16]. Experimental data obtained for the reaction (see $[15,17,18]$ ):

$$
d \uparrow+A_{t}=\pi^{ \pm}\left(0^{0}\right)+X,
$$

shown in Fig. 4.

From the figure it is clear that for more definite conclusions it is necessary to reduce errors if we use the same measurement setup as in $[15,17,18]$, see Fig. 5.

With this formulation, for the intensity of the discharge $I_{d \uparrow}=10^{11}$ burst $^{-1}$ and the carbon target $l_{t}=10 \mathrm{~g} / \mathrm{cm}^{2}$ to obtain the data shown in Fig. 5. with three times less error, the accelerator week is required. 


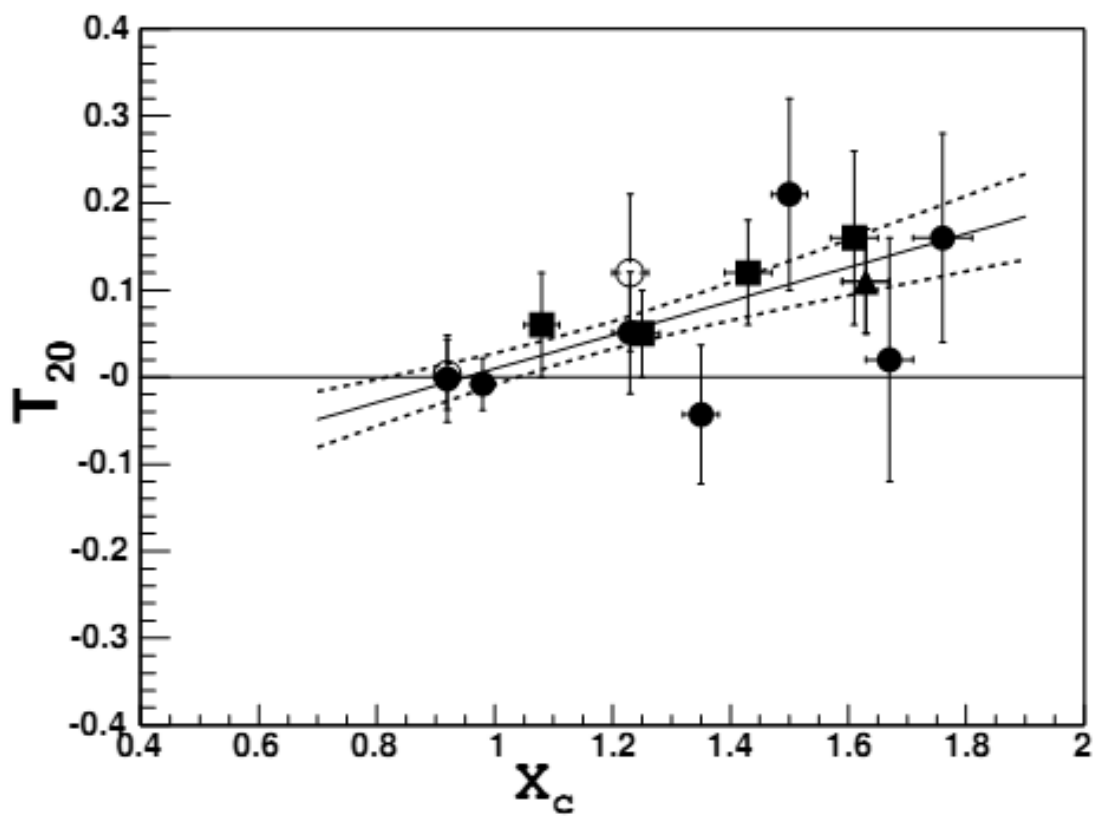

Figure 4. Dependence on the cumulative number $X_{c}$ (see [19]) of the analyzing power of $T_{20}$ for the reaction (8): open circles $-\pi^{+} ; A_{t}=12$, closed circles $-\pi^{-} ; A_{t}=12$, closed triangles $-\pi^{+} ; A_{t}=12$, closed squares $-\pi^{-} ; A_{t}=1$, the solid line is the result of fitting with a linear dependence with the errors shown by dashed lines

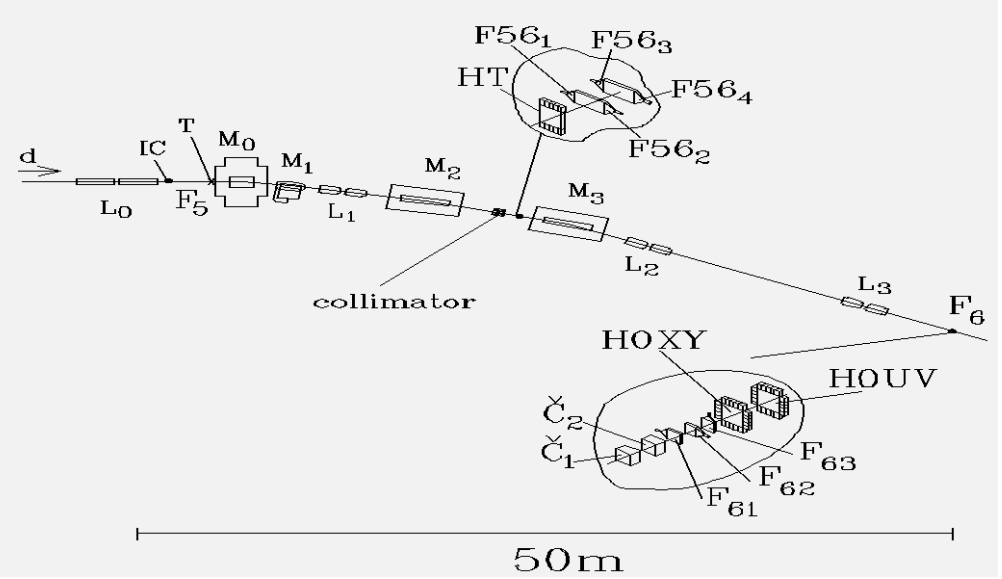

Figure 5. The scheme of measuring $T_{20}$ on channel 4B of the LHEP accelerator complex JINR. The target is marked with T, IC-ionization chamber for beam monitoring, $\mathrm{M}_{i}$ - deflecting magnets, $\mathrm{L}_{i}$ doublets of quadrupole lenses, $\mathrm{F}_{i j}$-scintillation counters, $\check{\mathrm{C}}_{i}$-Cherenkov threshold counters and HOXY, HOUV - scintillation hodoscopes 


\section{Conclusion}

Here, we list the main conclusions of the work:

1. It is shown that data on the asymmetry of the production of charged pions by transversely polarized protons can be obtained on MPD using beam of polarized protons.

2. It is shown that data on the asymmetry of pion production by polarized deuterons and protons can help to obtain data on the asymmetry of the production of pions by polarized neutrons.

3. It is shown that at the LPHE JINR accelerator complex in a beam of tensor-polarized deuterons, it is possible to obtain the experimental data on the tensor analyzing $T_{20}$ with three times less errors than previous data during one week at the accelerator.

The work did not discuss the possibility of measuring the asymmetry of neutral pion production at MPD. Of particular interest for such measurements are data on the asymmetry of the production of neutral pions by polarized neutrons. For measurements on an internal target, it is possible to expand the measurement program in the scheme discussed in the article by including studies of the tensor analyzing power for the reaction (8) $A_{y y}$ with the pion production at a nonzero angle (see [20]).

\section{References}

[1] http://nica.jinr.ru/complex.php

[2] http://nica.jinr.ru/ru/

[3] http://nuclphys.sinp.msu.ru/conf/epp10/Meshkov.pdf

[4] D.L. Adams et al., Phys. Lett. B 264, 462 (1991)

[5] D.L. Adams et al., Z. Phys. C 56, 181 (1992)

[6] STAR Collaboration, arXiv:hep-exp 1708.07080 (2017)

[7] Allgower C.E. et al., Phys.Rev. D 65, 092008 (2002)

[8] Mauro Anselmino, M.E. Boglione, F. Murgia, arXiv:hep-ph/9410382 (1994)

[9] http://mpd.jinr.ru/mpd/

[10] V.S. Barashenkov, N.V. Slavin, PEPAN 15, 997 (1984)

[11] A.N. Vasiliev and V.V. Mochalov, Phys. of Atomic Nuclei 67, 216 (2004)

[12] R.V. Reid Ann.Phys. (N.Y.) 50, 411 (1968)

[13] A. Litvinenko, E. Litvinenko Physics of Atomic Nuclei 78(2), 337 (2015)

[14] L. Zolin et al., Nucl. Phys. A 689, 414c (2001)

[15] S.V. Afanasiev et al., Nucl.Phys. A 625, 817 (1997)

[16] A.Yu. Illarionov, A.G. Litvinenko, G.I. Lykasov, Eur. Phys. J. A 14, 247(2002)

[17] S.V. Afanasiev et al., Phys. Lett. B 445, 14-19 (1998)

[18] S. Afanasiev et al., Nuclear Physics A 721, 645c-648c (2003)

[19] A.G. Litvienko, A.I. Malakhov, P.I. Zarubin, PEPAN Lett. 1[93], 27 (1993)

[20] L. Zolin et al., Nucl. Phys. A 689, 414c (2001) 